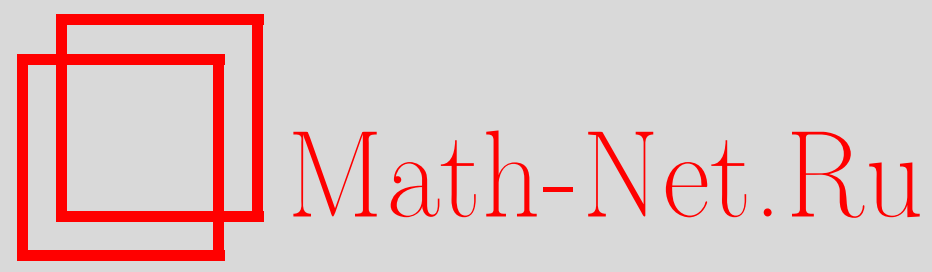

Е. И. Бережной, Подпространство $C[0,1]$, состоящее из функций, не имеющих конечных одностороних производных ни в одной точке, Матем. заметки, 2003, том 73, выпуск $3,348-354$

DOI: https://doi.org/10.4213/mzm191

Использование Общероссийского математического портала Math-Net.Ru подразумевает, что вы прочитали и согласны с пользовательским соглашением http://www . mathnet.ru/rus/agreement

Параметры загрузки:

IP : 3.91 .87 .62

26 апреля 2023 г., 13:46:27

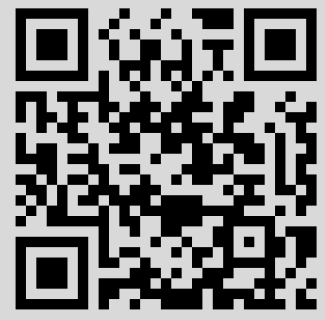




\section{ПОДПРОСТРАНСТВО $C[0,1]$, СОСТОЯЩЕЕ ИЗ ФУНКЦИЙ, НЕ ИМЕЮЩИХ КОНЕЧНЫХ ОДНОСТОРОНИХ ПРОИЗВОДНЫХ НИ В ОДНОЙ ТОЧКЕ}

\section{Е.И. Бережной}

Построено замкнутое бесконечномерное подпространство $G \subset C([0,1])$, дающее утвердительный ответ на старый вопрос: существует ли бесконечномерное замкнутое подпространство $G \subset C[0,1]$ такое, что каждая отличная от тожсдественного нуля функиия $y \in G$ не имеет ни в одной точке ни правой, ни левой конечной производной.

Библиографоял: 5 названий.

В геометрической теории банаховых пространств значительный интерес представляет следующая задача (на нее обращалось внимание в [1]).

Существует ли бесконечномерное замкнутое подпространство $Y \subset C[0,1]$ такое, что каждая отличная от тохдественного нуля функиия $y \in Y$ не имеет ни в одной точке ни правой, ни левой конечной производной.

Этим вопросом занимались многие математики [1]-[5]. Последнее достижение выглядит следующим образом.

TEOPEмA FGK [1]. Существует замкнутое бесконечномерное подпространство $E \subset C[0,1]$ и подмножсество $A$ отрезка $[0,1]$ лебеговой меры единица такое, что каждая отличная от тождественного нуля функиия $x \in E$ нигде не дифферениируема на $(0,1)$ и не имеет ни в одной точке множества $A$ ни правой, ни левой конечной производной.

Основным результатом настоящей статьи является окончательное решение задачи, приведенной в начале работы.

ТЕорема. Существует замкнутое бесконечномерное подпространство $G \subset$ $C([0,1])$ такое, что каждая отличная от тождественного нуля функиия $x \in G$ не имеет ни в одной точке $(0,1)$ ни правой, ни левой конечной производной.

Для доказательства основной теоремы нам потребуются некоторые предварительные построения.

Предположим сначала, что построены две бесконечные верхнетреугольные матрицы $D$ и $H$

$$
D=\left(\begin{array}{cccc}
d_{1,1} & d_{1,2} & d_{1,3} & \ldots \\
0 & d_{2,2} & d_{2,3} & \ldots \\
0 & 0 & d_{3,3} & \ldots \\
\ldots & \ldots & \ldots & \ldots
\end{array}\right), \quad H=\left(\begin{array}{cccc}
n_{1,1} & n_{1,2} & n_{1,3} & \ldots \\
0 & n_{2,2} & n_{2,3} & \ldots \\
0 & 0 & n_{3,3} & \ldots \\
\ldots \ldots & \ldots & \ldots
\end{array}\right)
$$

Работа выполнена при финансовой поддержке Российского фонда фундаментальных исследований, грант № 02-01-00428. 
причем элементы $\left\{d_{j, k}\right\}$ при всех $j, k \in \mathbb{N}$ и $k \geqslant j$ являются положительными числами, а элементы $\left\{n_{j, k}\right\}$ при всех $j, k \in \mathbb{N}$ и $k \geqslant j$ являются натуральными числами.

Пусть, кроме того, для элементов матрищ $D$ и $H$ вьполнены соотношения:

для любых $j, k \in \mathbb{N}$ c $k>j$ справедливо неравенство

$$
d_{j, k}>2^{3+k} d_{j+1, k}
$$

для любых $j, k \in \mathbb{N}$ с $k \geqslant j$ справедливо неравенство

$$
d_{j, k+1}>2^{3} d_{j, k}
$$

для любых чисел $j, k \in \mathbb{N} c k \geqslant j$ справедливы соотношения

$$
\frac{n_{k+1, k+1}}{8 n_{1, k}} \in \mathbb{N}, \quad \frac{n_{j, k}}{8 n_{j+1, k}} \in \mathbb{N},
$$

т.е. эти числа являются натуральными;

для любого фиксированного $j \in \mathbb{N}$ справедливы неравенства

$$
\frac{1}{2}<\frac{d_{j, j}}{n_{j, j}}<1
$$

для любого фиксированного $j$ при всех $k=j, j+1, j+2, \ldots$ справедливы неравенства

$$
\frac{d_{j, k+1}}{n_{j, k+1}} \leqslant \frac{1}{8} \frac{d_{j, k}}{n_{j, k}}
$$

Матрицу $D$ будем назьвать матрицей производных, а матрицу $H$ будем назьвать матрицей периодов. Для элементов матрищы $H$ положим $h_{j, k}=1 / n_{j, k}, j=1,2, \ldots$, $k=j, j+1, j+2, \ldots$.

По матрице периодов $H$ построим систему узлов, полагая $\tau_{j, k, l}=l h_{j, k}, l \in \mathbb{Z}$.

Для $t \in[0,1]$ положим $\varphi_{0}(t)=0,25-|t-0,5|$ и доопределим функцию $\varphi_{0}$ на всю ось периодически с периодом единица.

ЛЕмма 1. Для любых $t, s \in \mathbb{R} u j, k \in \mathbb{N}, k \geqslant j$, справедливо неравенство

$$
\left|\frac{1}{n_{j, k}}\left\{\varphi_{0}\left(n_{j, k} t\right)-\varphi_{0}\left(n_{j, k} s\right)\right\}\right| \leqslant|t-s| ;
$$

если $\tau_{j, k, l} \leqslant t, s \leqslant \tau_{j, k, l+1}$, то справедливо равенство

$$
\left|\frac{1}{n_{j, k}}\left\{\varphi_{0}\left(n_{j, k} t\right)-\varphi_{0}\left(n_{j, k} s\right)\right\}\right|=|t-s| ;
$$

если

$$
t<\tau_{j, k, l}<t+h_{k+1, k+1}
$$

то справедливо неравенство

$$
\left|\frac{1}{n_{j, k}}\left\{\varphi_{0}\left(n_{j, k}\left(t+\frac{h_{1, k}}{2}\right)\right)-\varphi_{0}\left(n_{j, k} t\right)\right\}\right| \geqslant \frac{1}{4} h_{1, k} .
$$


ДоКАЗАТЕЛЬСТво. Соотношения (6) и (7) очевидны.

Докажем (9). Из условий (3) и (8) следует, что $h_{1, k} / 2+t \in\left[\tau_{j, k, l}, \tau_{j, k, l+1}\right]$. Поэтому (9) следует из $(3),(6),(7)$ и цепочки неравенств

$$
\begin{aligned}
& \left|\frac{1}{n_{j, k}}\left\{\varphi_{0}\left(n_{j, k}\left(t+\frac{h_{1, k}}{2}\right)\right)-\varphi_{0}\left(n_{j, k} t\right)\right\}\right| \\
& \geqslant\left|\frac{1}{n_{j, k}}\left\{\varphi_{0}\left(n_{j, k}\left(t+\frac{h_{1, k}}{2}\right)\right)-\varphi_{0}\left(n_{j, k} \tau_{j, k, l}\right)\right\}\right| \\
& \quad-\left|\frac{1}{n_{j, k}}\left\{\varphi_{0}\left(n_{j, k} \tau_{j, k, l}\right)-\varphi_{0}\left(n_{j, k} t\right)\right\}\right| \\
& \geqslant\left|t+\frac{h_{1, k}}{2}-\tau_{j, k, l}\right|-\left|\tau_{j, k, l}-t\right| \geqslant \frac{h_{1, k}}{2}-h_{k+1, k+1}-h_{k+1, k+1} \\
& =\frac{h_{1, k}}{2}-2 h_{k+1, k+1}=\frac{h_{1, k}}{2}\left(1-4 \frac{h_{k+1, k+1}}{h_{1, k}}\right) \geqslant \frac{1}{4} h_{1, k} .
\end{aligned}
$$

Лемма полностью доказана.

Для каждого $j \in \mathbb{N}$ с помощью матриц $D, H$ по функции $\varphi_{0}(t)$ построим новые функции

$$
\psi_{j}(t)=\sum_{l=j}^{\infty} d_{j, l} h_{j, l} \varphi_{0}\left(n_{j, l} t\right)
$$

Для $k>j$ положим

$$
\psi_{j, k}^{1}(t)=\sum_{l=j}^{k} d_{j, l} h_{j, l} \varphi_{0}\left(n_{j, l} t\right), \quad \psi_{j, k}^{2}(t)=\sum_{l=k+1}^{\infty} d_{j, l} h_{j, l} \varphi_{0}\left(n_{j, l} t\right) .
$$

Легко видеть, что для всех $k \geqslant j$ справедливо равенство

$$
\psi_{j}(t)=\psi_{j, k}^{1}(t)+\psi_{j, k}^{2}(t) .
$$

Сразу же отметим, что из (3) следуют соотношения

$$
\begin{gathered}
\frac{h_{j, j+k}}{h_{j, j+k+1}} \in \mathbb{N}, \\
\frac{h_{j, j+k}}{h_{j, j+k+1}}=\frac{n_{j, j+k+1}}{n_{j, j+k}}=\frac{n_{j, j+k+1}}{n_{j+1, j+k+1}} \frac{n_{j+1, j+k+1}}{n_{j+2, j+k+1}} \cdots \frac{n_{j+k+1, j+k+1}}{n_{1, j+k}} \frac{n_{1, j+k}}{n_{2, j+k}} \cdots \frac{n_{j-1, j+k}}{n_{j, j+k}} \\
\geqslant 8^{k+1+j} .
\end{gathered}
$$

Поэтому период функции $\psi_{j, k}^{2}(t)$ равен $h_{j, k+1}$ и, следовательно, согласно $(3)$ периодами этой функции будут числа $h_{1, k} / 2$ и $h_{k+1, k+1}$.

ЛЕмма 2. Пусть заданы натуральные числа $j, k$ c $k>j$. Если при некотором $l$ верны неравенства $\tau_{j, k, l} \leqslant t<t+h_{k+1, k+1} \leqslant \tau_{j, k, l+1}$, то справедливо неравенство

$$
\left|\psi_{j}\left(t+h_{k+1, k+1}\right)-\psi_{j}(t)\right| \geqslant \frac{3}{4} d_{j, k} h_{k+1, k+1} .
$$

Если при некотором $l$ верны неравенства $t<\tau_{j, k, l}<t+h_{k+1, k+1}$, то справедливо неравенство

$$
\left|\psi_{j}\left(t+\frac{h_{1, k}}{2}\right)-\psi_{j}(t)\right| \geqslant \frac{1}{4} d_{j, k} \frac{h_{1, k}}{2} .
$$


ДокАЗАТЕЛЬство. Докажем (11). Из представления (10) и периодичности функции $\psi_{j, k}^{2}(t)$ с периодом $h_{k+1, k+1}$ получим

$$
\begin{aligned}
\left|\psi_{j}\left(t+h_{k+1, k+1}\right)-\psi_{j}(t)\right|= & \left|\psi_{j, k}^{1}\left(t+h_{k+1, k+1}\right)-\psi_{j, k}^{1}(t)\right| \\
= & \left|\sum_{l=j}^{k} d_{j, l} h_{j, l} \varphi_{0}\left(n_{j, l}\left(t+h_{k+1, k+1}\right)\right)-\sum_{l=j}^{k} d_{j, l} h_{j, l} \varphi_{0}\left(n_{j, l} t\right)\right| \\
\geqslant & d_{j, k} h_{j, k}\left|\varphi_{0}\left(n_{j, k}\left(t+h_{k+1, k+1}\right)\right)-\varphi_{0}\left(n_{j, k} t\right)\right| \\
& -\sum_{l=j}^{k-1} d_{j, l} h_{j, l}\left|\varphi_{0}\left(n_{j, l}\left(t+h_{k+1, k+1}\right)\right)-\varphi_{0}\left(n_{j, l} t\right)\right| .
\end{aligned}
$$

Далее применим равенство (7) из леммы 1 и условие (2). Тогда получим

$$
\left|\psi_{j}\left(t+h_{k+1, k+1}\right)-\psi_{j}(t)\right| \geqslant d_{j, k} h_{k+1, k+1}-h_{k+1, k+1} \sum_{l=j}^{k-1} d_{j, l} \geqslant \frac{3}{4} h_{k+1, k+1} d_{j, k} .
$$

Неравенство (11) доказано.

Для доказательства (12) нужно действовать аналогично, используя неравенство (9) из леммы 1.

Лемма полностью доказана.

Положим $G=\overline{\operatorname{span}\left\{\psi_{i} /\left\|\psi_{i} \mid C[0,1]\right\|\right\}_{i=1}^{\infty}}$.

Лемма 3. Пространство $G$ является замкнутым подпространством пространства $C[0,1]$, изоморфным пространству $\ell^{1}$, причем базис $\left\{\psi_{i} /\left\|\psi_{i} \mid C[0,1]\right\|\right\}_{i=1}^{\infty}$ пространства $G$ әквивалентен единичному базису пространства $\ell^{1}$.

ДокАЗАТЕЛЬСтво. Во-первых, покажем, что при всех $i \in \mathbb{N}$ верны неравенства

$$
\frac{6}{7} d_{i, i} h_{i, i} \leqslant\left\|\psi_{i} \mid C[0,1]\right\| \leqslant \frac{8}{7} d_{i, i} h_{i, i} .
$$

Действительно, из (5) следует, что

$$
\left\|\psi_{i} \mid C[0,1]\right\| \leqslant \sum_{l=i}^{\infty} d_{i, l} h_{i, l} \leqslant d_{i, i} h_{i, i}\left(1+\frac{1}{8}+\left(\frac{1}{8}\right)^{2}+\cdots\right)=\frac{8}{7} d_{i, i} h_{i, i}
$$

и

$$
\begin{aligned}
\left\|\psi_{i} \mid C[0,1]\right\| & \geqslant\left\|d_{i, i} h_{i, i} \varphi_{0}\left(n_{i, i} t\right)\left|C[0,1]\|-\| \sum_{l=i+1}^{\infty} d_{i, l} h_{i, l} \varphi_{0}\left(n_{i, l} t\right)\right| C[0,1]\right\| \\
& \geqslant d_{i, i} h_{i, i}-\sum_{l=i+1}^{\infty} d_{i, l} h_{i, l} \geqslant d_{i, i} h_{i, i}\left(1-\frac{1}{7}\right) .
\end{aligned}
$$

Положим $e^{i}(t)=\psi_{i}(t) /\left\|\psi_{i} \mid C[0,1]\right\|$ и покажем, что для любого $k \in \mathbb{N}$ и любого набора чисел $\left\{a_{i}\right\}$ верны неравенства

$$
\frac{5}{16} \sum_{i=1}^{k}\left|a_{i}\right| \leqslant\left\|\sum_{i=1}^{k} a_{i} e^{i}\left|C[0,1] \| \leqslant \sum_{i=1}^{k}\right| a_{i} \mid\right.
$$


Правое неравенство в (14) очевидно.

Перейдем к доказательству левого неравенства в (14). Зафиксируем $k \in \mathbb{Z}$ и образуем множества

$$
\begin{aligned}
& D(i, k)_{+}=\left\{t \in\left[\tau_{i, i, k}, \tau_{i, i, k+1}\right]: \varphi_{0}\left(n_{i, i} t\right) \geqslant \frac{1}{2}\right\}, \\
& D(i, k)_{-}=\left\{t \in\left[\tau_{i, i, k}, \tau_{i, i, k+1}\right]: \varphi_{0}\left(n_{i, i} t\right) \leqslant-\frac{1}{2}\right\} .
\end{aligned}
$$

Напомним, что $\left\{\tau_{i, i, k}\right\}$ есть система узлов, построенная по матрице $H$ для функции $\varphi_{0}\left(n_{i, i} t\right)$.

Из рассмотрения графика функции $\varphi_{0}\left(n_{i, i} t\right)$ легко видеть, что множества $D(i, k)_{+}$,

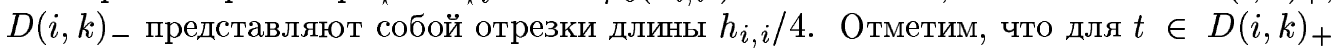
выполняется неравенство

$$
\psi_{i}(t) \geqslant d_{i, i} h_{i, i} \varphi_{0}\left(n_{i, i} t\right)-\sum_{l=i+1}^{\infty} d_{i, l} h_{i, l} \geqslant d_{i, i} h_{i, i}\left(\frac{1}{2}-\frac{1}{7}\right)=\frac{5}{14} d_{i, i} h_{i, i}
$$

а для $t \in D(i, k)$ _ вьполняется неравенство

$$
\psi_{i}(t) \leqslant d_{i, i} h_{i, i} \varphi_{0}\left(n_{i, i} t\right)+\sum_{l=i+1}^{\infty} d_{i, l} h_{i, l} \leqslant-d_{i, i} h_{i, i}\left(\frac{1}{2}-\frac{1}{7}\right)=-\frac{5}{14} d_{i, i} h_{i, i} .
$$

Из соотношений (3) следует, что

$$
\begin{gathered}
\frac{h_{i, i}}{h_{i+1, i+1}} \in \mathbb{N} \\
\frac{h_{i, i}}{h_{i+1, i+1}}=\frac{n_{i+1, i+1}}{n_{i, i}}=\frac{n_{i+1, i+1}}{n_{1, i}} \frac{n_{1, i}}{n_{2, i}} \cdots \frac{n_{i-1, i}}{n_{i, i}} \geqslant 8^{i}
\end{gathered}
$$

Поэтому каждый из отрезков $D(i, k)_{+}, D(i, k)_{\text {_ }}$ содержит не менее двух периодов функции $e^{i+1}(t)$.

Теперь у нас все готово для доказательства левого неравенства в (14). Без ограничения общности можно считать, что $a_{1} \geqslant 0$. Положим $T_{1}=D(1,0)_{+}$. Если $a_{2} \geqslant 0$, то найдется отрезок $T_{2}=D\left(2, l_{2}\right)_{+} \subset T_{1}$, если же $a_{2}<0$, то найдется отрезок $T_{2}=D\left(2, l_{2}\right)_{-}$ $\subset T_{1}$; если $a_{3} \geqslant 0$, то найдется отрезок $T_{3}=D\left(3, l_{3}\right)_{+} \subset T_{2}$, если же $a_{3}<0$, то найдется отрезок $T_{3}=D\left(3, l_{3}\right)_{-} \subset T_{2}$ и т.д. Из построения, (13) и $(15),(16)$ следует, что для всех $t \in T_{k}$ вьполнено соотношение

$$
\begin{aligned}
\left|\sum_{i=1}^{k} a_{i} e^{i}(t)\right| & =\sum_{i=1}^{k}\left|a_{i}\right| \cdot\left|e^{i}(t)\right|=\sum_{i=1}^{k}\left|a_{i}\right| \frac{\left|\psi_{i}(t)\right|}{\left\|\psi_{i} \mid C[0,1]\right\|} \\
& \geqslant \frac{5}{14} \sum_{i=1}^{k}\left|a_{i}\right| \frac{d_{i, i} h_{i, i}}{\left\|\psi_{i} \mid C[0,1]\right\|} \geqslant\left(\frac{5}{14}: \frac{8}{7}\right) \sum_{i=1}^{k}\left|a_{i}\right|=\frac{5}{16} \sum_{i=1}^{k}\left|a_{i}\right| .
\end{aligned}
$$

Таким образом, и левое неравенство в (14) доказано. Из (14) непосредственно следует утверждение леммы.

Лемма доказана. 
Лемма 4. Пусть построены матрицы $D$ и $H$, удовлетворяющие (1)-(5). Тогда любая отличная от тождественного нуля функиия из $G$ не имеет ни в одной точке $(0,1)$ ни правой, ни левой конечной производной.

ДокАЗАтЕльство. Достаточно показать, что любая функция

$$
f(t)=\sum_{i=j}^{\infty} a_{i} \psi_{i}(t)
$$

с $a_{j} \neq 0$, для которой

$$
\sum_{i=j}^{\infty}\left|a_{i}\right| \leqslant 1
$$

ни в одной точке не имеет ни правой, ни левой конечной производной.

Покажем, что ни в одной точке сегмента $[0,1)$ данная функция не имеет конечной правой производной.

Зафиксируем $t_{0} \in[0,1)$. При каждом $k \in \mathbb{N}, k>j$, для которого $h_{k, k}<1-t_{0}$, рассмотрим точки $t_{0}, t_{0}+h_{k+1, k+1}$. У нас имеются две возможности.

Найдется индекс $l$ такой, что эти точки лежат между узлами набора $\tau_{j, k, l}$, т.е.

$$
t_{0}, t_{0}+h_{k+1, k+1} \in\left[\tau_{j, k, l}, \tau_{j, k, l+1}\right]
$$

или найдется индекс $l$ такой, что справедливо неравенство

$$
t_{0}<\tau_{j, k, l}<t_{0}+h_{k+1, k+1} .
$$

Рассмотрим первую возможность. Сначала отметим, что согласно (3) при всех $l=$ $k+1, k+2, \ldots$ верно соотношение

$$
\psi_{l}\left(t+h_{k+1, k+1}\right) \equiv \psi_{l}(t)
$$

Кроме того, из (3) следует, что при всех $i=j, j+1, \ldots, k$ верно соотношение

$$
\psi_{i, k}^{2}\left(t+h_{k+1, k+1}\right) \equiv \psi_{i, k}^{2}(t) .
$$

Поэтому

$$
f\left(t+h_{k+1, k+1}\right)-f(t)=\sum_{i=j}^{k} a_{i}\left\{\psi_{i, k}^{1}\left(t+h_{k+1, k+1}\right)-\psi_{i, k}^{1}(t)\right\} .
$$

Следовательно, справедлива оценка

$$
\begin{aligned}
\left|f\left(t_{0}+h_{k+1, k+1}\right)-f\left(t_{0}\right)\right| \geqslant & \left|a_{j}\left\{\psi_{j, k}^{1}\left(t_{0}+h_{k+1, k+1}\right)-\psi_{j, k}^{1}\left(t_{0}\right)\right\}\right| \\
& -\sum_{i=j+1}^{k}\left|a_{i}\right| \cdot\left|\psi_{i, k}^{1}\left(t_{0}+h_{k+1, k+1}\right)-\psi_{i, k}^{1}\left(t_{0}\right)\right| \\
= & A_{1}-A_{2} .
\end{aligned}
$$

Для оценки $A_{1}$ воспользуемся леммой 2:

$$
A_{1}=\left|a_{j}\right| \cdot\left|\psi_{j, k}^{1}\left(t_{0}+h_{k+1, k+1}\right)-\psi_{j, k}^{1}\left(t_{0}\right)\right| \geqslant\left|a_{j}\right| \frac{3 d_{j, k}}{4} h_{k+1, k+1} .
$$


Для оценки $A_{2}$ воспользуемся леммой 1 и соотношениями $(1),(2)$ и $(17)$ :

$$
\begin{aligned}
A_{2} & =\sum_{i=j+1}^{k}\left|a_{i}\right| \cdot\left|\psi_{i, k}^{1}\left(t_{0}+h_{k+1, k+1}\right)-\psi_{i, k}^{1}\left(t_{0}\right)\right| \\
& \leqslant h_{k+1, k+1} \sum_{i=j+1}^{k}\left|a_{i}\right| \sum_{l=i}^{k} d_{i, l} \leqslant \frac{8}{7} h_{k+1, k+1} \sum_{i=j+1}^{k}\left|a_{i}\right| d_{i, k} \\
& \leqslant \frac{8}{7} h_{k+1, k+1} \sum_{i=j+1}^{k} d_{i, k} \leqslant \frac{64}{49} h_{k+1, k+1} d_{j+1, k} .
\end{aligned}
$$

Из (20)-(22) с учетом соотношения (1) следует, что если найдется подпоследовательность $\left\{h_{k+1, k+1}\right\}_{k=j}^{\infty}$, удовлетворяющая (18), то в точке $t_{0}$ нет конечной правой производной.

Случай вьполнения соотношений (19) рассматривается аналогично, только нужно заменить $h_{k+1, k+1}$ на $h_{1, k} / 2$ и вместо неравенства (11) из леммы 2 использовать неравенство (12) из той же леммы.

Так как у последовательности $\left\{h_{k+1, k+1}\right\}_{k=j}^{\infty}$ обязательно найдется подпоследовательность, удовлетворяющая или (18), или (19), то у заданной функции нет в точке $t_{0}$ конечной правой производной.

Аналогично показьвается, что у функции $f$ ни в одной точке сегмента $(0,1]$ нет конечной левой производной.

Лемма доказана.

ДОКАЗАТЕЛЬСТВО оСНОВНОЙ ТЕОРЕМЫ. Продемонстируем конструкцию матриц $D$ и $H$. Выберем сначала $n_{1,1}$ произвольно. Затем выберем $d_{1,1}$ так, чтобы выполнялось условие (4). Затем выберем $n_{2,2}$ так, чтобы вьполнялось условие $(3)$, затем выберем $d_{2,2}$ так, чтобы вьполнялось условие $(4)$, потом выберем $d_{1,2}$ так, чтобы выполнялись условия (1), (2), потом выберем $n_{1,2}$ так, чтобы вьполнялись условия (3) и (5) и т.д.

Для окончания доказательства основной теоремы осталось воспользоваться леммой 4.

Теорема доказана.

\section{СПИСОК ЦИТИРОВАННОЙ ЛИТЕРАТУРЫ}

[1] Fonf V. P., Gurariy V. I., Kadets M. I. An infinite-dimensional subspace $C[0,1]$ consisting of nowhere differentiable functions // C. R. Acad. Bulg. Sci. 1999. V. 52. №11-12. P. 13-16.

[2] Зигмунд А. Тригонометрические ряды. Т. 1. М.: Мир, 1965.

[3] Петрушев П. П., Троянски С. Л. О теореме Банаха-Мазура об универсальности $C[0,1]$ // Comp. rend. Acad. Bulg. Sci. 1984. V. 37. № 3. P. 283-285.

[4] Гурарий В.И.Линейное пространство состоящее из всюду недифференцируемых функций // Comp. rend. Acad. Bulg. Sci. 1991. V. 44. № 5. P. 13-15.

[5] Rodriguez-Piazza I. Every separable Banach space is isometric to a space of continuous nowhere differentiable functions // Proc. Amer. Math. Soc. 1995. V. 123. № 12. P. 3650-3654.

Ярославский государственный универститет им. П.Г. Демидова

Поступило

E-mail: ber@uniyar.ac.ru

15.12 .2000

Исправленный вариант

14.12.2001 